\title{
Visual motion transforms visual space representations similarly throughout the human visual hierarchy
}

\author{
Ben M. Harvey ${ }^{\mathrm{a}, \mathrm{b}, *}$, Serge O. Dumoulin ${ }^{\mathrm{a}, 1}$ \\ a Experimental Psychology, Helmholtz Institute, Utrecht University, Utrecht, Netherlands \\ b Faculty of Psychology and Education Sciences, University of Coimbra, Coimbra, Portugal
}

\section{A R T I C L E I N F O}

\section{Article history:}

Received 4 June 2015

Accepted 29 November 2015

Available online 5 December 2015

\section{Keywords:}

Vision

Motion

7 T fMRI

Population receptive field

\begin{abstract}
A B S T R A C T
Several studies demonstrate that visual stimulus motion affects neural receptive fields and fMRI response amplitudes. Here we unite results of these two approaches and extend them by examining the effects of visual motion on neural position preferences throughout the hierarchy of human visual field maps. We measured population receptive field ( $\mathrm{pRF}$ ) properties using high-field fMRI $(7 \mathrm{~T})$, characterizing position preferences simultaneously over large regions of the visual cortex. We measured pRFs properties using sine wave gratings in stationary apertures, moving at various speeds in either the direction of pRF measurement or the orthogonal direction. We find direction- and speed-dependent changes in pRF preferred position and size in all visual field maps examined, including V1, V3A, and the MT + map T01. These effects on pRF properties increase up the hierarchy of visual field maps. However, both within and between visual field maps the extent of pRF changes was approximately proportional to $\mathrm{pRF}$ size. This suggests that visual motion transforms the representation of visual space similarly throughout the visual hierarchy. Visual motion can also produce an illusory displacement of perceived stimulus position. We demonstrate perceptual displacements using the same stimulus configuration. In contrast to effects on pRF properties, perceptual displacements show only weak effects of motion speed, with far larger speed-independent effects. We describe a model where low-level mechanisms could underlie the observed effects on neural position preferences. We conclude that visual motion induces similar transformations of visuo-spatial representations throughout the visual hierarchy, which may arise through low-level mechanisms.
\end{abstract}

(c) 2015 Elsevier Inc. All rights reserved.

\section{Introduction}

Visual motion can affect the visuo-spatial responses of neurons in the visual hierarchy. Motion can also affect the perceived position of visual stimuli (Whitney, 2002). For example, when moving patterns are presented within stationary apertures, aperture position appears displaced in the direction of motion (Ramachandran and Anstis, 1990; De Valois and De Valois, 1991).

These changes in perceived position likely result from changes in neural representations of visual space. Neural mechanisms acting at various levels of the visual hierarchy have been proposed to explain these perceptual effects. These mechanisms are not mutually exclusive. First, displacement of the receptive field by motion may affect perceived position. Support for this hypothesis is provided by direction selective cells in cat primary visual cortex (V1) that have their receptive field

\footnotetext{
* Corresponding author at: Faculty of Psychology and Education Sciences, University of Coimbra, Rua do Colégio Novo, Coimbra 3001-802, Portugal.

E-mail addresses: b.m.harvey@uu.nl (B.M. Harvey), s.o.dumoulin@uu.nl (S.O. Dumoulin).

${ }^{1}$ Experimental Psychology, Utrecht University, Heidelberglaan 2, Utrecht, 3584 CS, Netherlands.
}

preferred positions displaced against the direction of motion (Fu et al., 2004). This displacement is proposed to reflect asymmetries to the receptive field inputs in the representation of starting and continuing motions. Increased neural response amplitudes at the start of the motion trajectory may induce these asymmetries, and bias visual field map activation (Whitney et al., 2003; Liu et al., 2006; Maloney et al., 2014). Second, these perceptual effects may arise in specialized motion processing areas like MT + , where patterns of fMRI activation follow the perceived position of the stimuli rather than their retinal position (Fischer et al., 2011; Maus et al., 2013). Indeed, feedback connections from MT to V1 have been implicated in motion-induced changes in perceived position (De Valois and De Valois, 1991; Nishida and Johnston, 1999). Third, high-level mechanisms such as motion-dependent shifts in spatial attention have also been proposed (Baldo and Klein, 1995). Fourth, perceived position may change through predictive coding, so that the neural representation of position follows expectations from previous experience (Roach et al., 2011; Maloney et al., 2014; Schellekens et al., 2014). Finally, extraclassical effects may facilitate neural responses beyond the stimulus in the direction of motion and thereby cause direction-dependent changes in neural activation (Watamaniuk and McKee, 1995; Ledgeway and Hess, 2002; Ledgeway et al., 2005). 
Here we aim to unify these neurophysiological and fMRI results, and extend them by asking: how visuo-spatial response preferences are affected by motion in visual stimuli; where in the visual processing hierarchy these effects occur; and how they progress throughout the hierarchy. To do this, we characterize population receptive field (pRF) properties measured with different stimulus motion speeds and directions in several visual field maps, including V1, V3A and the MT + map T01 (Amano et al., 2009). We find direction-specific and speed-dependent changes in pRF properties in all visual field maps examined, with effects increasing up the visual hierarchy. Across visual field maps and within visual field maps, the extent of pRF changes was approximately proportional to pRF size. We propose a model in which these changes in pRF properties may straightforwardly result from larger response amplitudes to appearing than continuing motions, consistent with established changes in response amplitude along the motion trajectory. We also demonstrate perceptual displacements using the same stimulus configuration, though these displacements do not change strongly with motion speed. Therefore, motion transforms the neural representation of visual space similarly throughout the visual processing hierarchy. These effects on the neural representation of visual space may underlie motion's effects on position perception, though aspects of neural and perceptual effects differ.

\section{Methods}

\section{Subjects}

Four subjects participated in the fMRI experiment (one female, age range 25-44 years). All subjects had normal or corrected-to-normal visual acuity. All experimental procedures were cleared by the medical ethics committee of University Medical Center Utrecht.

\section{PRF mapping stimuli}

PRF mapping stimuli were generated in Matlab (Mathworks, Natick, MA, USA) using the PsychToolbox (Brainard, 1997; Pelli, 1997). Visual stimuli were gamma-corrected (using the output of a PR650 colorimeter, Photo Research Inc., Chatsworth, CA, USA) and presented by backprojection onto a $15.0 \times 7.9 \mathrm{~cm}$ screen inside the MRI bore. The subject viewed the display through prisms and mirrors, and the total distance from the subject's eyes (in the scanner) to the display screen was $41 \mathrm{~cm}$. This gave a visual angle of $11^{\circ}$ for the vertical (smallest) dimension of the display. Visible display resolution was $1024 \times 538$ pixels.

The pRF mapping paradigm was similar to that described in previous studies (Dumoulin and Wandell, 2008; Amano et al., 2009; Levin et al., 2010; Winawer et al., 2010; Harvey and Dumoulin, 2011; Hoffmann et al., 2012; Zuiderbaan et al., 2012). The stimulus (Fig. 1A) consisted of bar-apertures at various orientations stepping across the visual field. The stimulus had a radius of $5.5^{\circ}$. After every volume acquisition (TR, $1500 \mathrm{~ms}$ ), the bar-aperture stepped $0.55^{\circ}$ across this visual field. So the bar-aperture took 20 TRs (30 s) to cross the stimulus area. The bar-aperture passed through the stimulus area alternating between cardinal and diagonal directions, with a 20 TR (30 s) blank display period following each cardinal direction bar pass (Fig. 1B).

We presented all stimuli at 99\% Michelson contrast, the maximum possible with the display. The contrast of the bar-aperture faded at its edges following a $1^{\circ}$ wide raised cosine to avoid hard edges that would increase the range of spatial frequencies present in the stimulus. Including these edges, the bar-aperture was $2^{\circ}$ wide. Within this bar-aperture, we showed a sine wave grating with a spatial frequency of 1 cycle $/{ }^{\circ}$ (Fig. 1A). We measured pRFs across four runs, in which gratings moved at one of four speeds $\left(1.25^{\circ} / \mathrm{s}, 2.5^{\circ} / \mathrm{s}, 3.75^{\circ} / \mathrm{s}\right.$ and $\left.5^{\circ} / \mathrm{s}\right)$ in the direction of pRF measurement (using a grating with the same orientation as the bar-aperture) (see Experimental rationale Section). To determine the direction-specificity of these effects, we also measured pRFs while gratings moved at two speeds $\left(2.5^{\circ} / \mathrm{s}\right.$ and $\left.5^{\circ} / \mathrm{s}\right)$ orthogonal to the direction of pRF measurement (using an orthogonally oriented grating). We measured responses to different speeds and directions in separate scanning runs in random order during the same session.

During each scanning run, subjects fixated a dot $\left(0.125^{\circ}\right.$ radius $)$ in the center of the display. This changed colors between red and green at random intervals. To ensure fixation and attention here, subjects pressed a button every time the color changed, on average every $3 \mathrm{~s}$ with a minimum change interval of 1.8 s. Subjects reported over $85 \%$ of these changes on every scanning run.

Because a moving pattern sometimes passed behind the fixation dot, we wanted to avoid involuntary motion tracking eye movements that would affect pRF parameter estimates. The fixation dot was surrounded by a white annulus (to $0.19^{\circ}$ radius) to increase the contrast here. This was then surrounded by a mean luminance gray annulus (the same

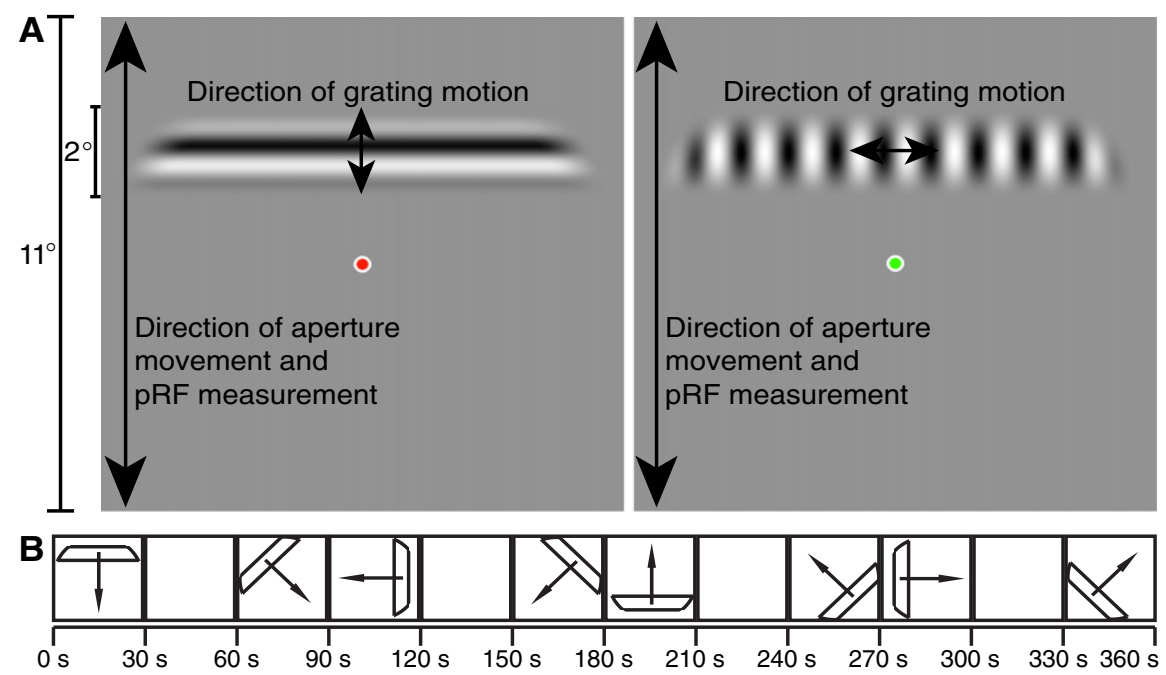

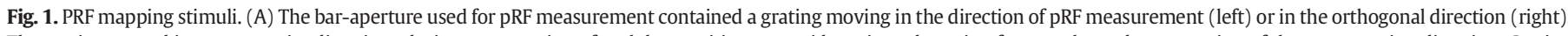

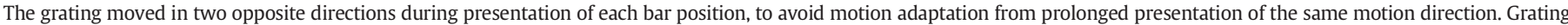

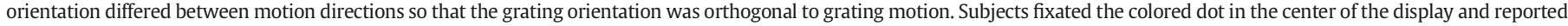

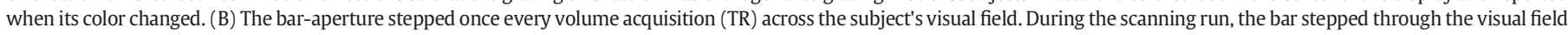
in eight directions, with each cardinal direction followed by a $30 \mathrm{~s}$ period ( $20 \mathrm{TRs}$ ) with no bar presented, allowing the fMRI signal to return to baseline. 
color as the stimulus background), occluding the stimulus to $0.5^{\circ}$ eccentricity. In tests before scanning, we found this design very effective in eliminating tracking eye movements.

\section{Eye tracking}

To ensure that different pRF properties at different speeds did not result from different motion-induced eye movements, we measured our subject's eye positions outside the scanner while watching the pRF mapping stimulus using a highly-accurate Eyelink II system (SR Research, Mississauga, Ontario, Canada). As we have demonstrated before (Harvey and Dumoulin, 2011; Dumoulin et al., 2014), the spread of gaze positions during moving bar presentations and the mean-luminance blank periods were not significantly different, and so presentation of stimulus motion did not induce eye movements. Furthermore, the spread of gaze positions in the direction of pRF measurement did not differ from the spread in the orthogonal direction, so direction-specific effects did not result from direction specific change in gaze position (Dumoulin et al., 2014). Finally, the spread of gaze positions in the direction of pRF measurement was not affected by the direction or speed of grating motion, so speed-dependent effects did not result from speeddependent changes in eye movements. In all subjects and stimulus conditions, the standard deviation of gaze positions around fixation was within $0.25^{\circ}$.

\section{MRI acquisition}

Anatomical MRI data were acquired on a Philips Achieva 3 T scanner (Philips Healthcare, Best, Netherlands) with a Quasar Dual gradient set. T1-weighted anatomical MRI data were acquired at a resolution of $0.75 \times 0.75 \times 0.8 \mathrm{~mm}$. Repetition time (TR) was $10.029 \mathrm{~ms}$, echo time (TE) was $4.6 \mathrm{~ms}$, and flip angle was $8^{\circ}$.

Functional $\mathrm{T} 2 *$-weighted 2D echo planar images were acquired on a Phillips Achieva $7 \mathrm{~T}$ scanner using a 16 channel head coil (Nova Medical, Wilmington, MA, USA) at a resolution of $1.98 \times 1.98 \times 2.00 \mathrm{~mm}$, with an field of view of $190 \times 190 \times 50 \mathrm{~mm}$. TR was $1500 \mathrm{~ms}$, TE was $25 \mathrm{~ms}$, and flip angle was $80^{\circ}$. Functional runs were each 248 time frames (372 s) in duration, of which the first eight time frames (12 s) were discarded to ensure the signal was at steady state. Four repeated runs were acquired for each stimulus condition and averaged together for analysis, with runs for the different stimulus conditions acquired interleaved in randomlyordered blocks. Each block contained all seven stimulus conditions and took one scanning session to complete.

\section{Preprocessing of anatomical and functional images}

Functional MRI analysis was performed in the mrVista software package, which is freely available at (http://white.stanford.edu/ software/). T1-weighted anatomical scans were automatically segmented using Freesurfer (Dale et al., 1999) and then hand-edited to minimize segmentation errors (Teo et al., 1997) using ITK-SNAP (Yushkevich et al., 2006). The cortical surface was reconstructed at the gray-white matter border and rendered as a smoothed 3D surface (Wandell et al., 2000). Head movement and motion artifacts between and within functional scans were measured and corrected for (Nestares and Heeger, 2000). Functional data were aligned to the anatomical scans (Nestares and Heeger, 2000) and interpolated to the anatomical segmentation space. Data from all sessions was imported into the same anatomical segmentation space and runs with the same stimulus condition were then averaged together.

Draining veins can spatially and temporally distort the fMRI signal around them (Olman et al., 2007; Winawer et al., 2010). We identified these locations by the mean signal intensity of the BOLD signal (Winawer et al., 2010; Harvey and Dumoulin, 2011; Harvey et al., 2013) and excluded these from further analysis. This procedure excludes regions where pRF parameters are unreliable reflections of neural response properties. However, similar results were found if these vein regions are included in the analysis.

\section{Population receptive field modeling}

Visual field mapping data were analyzed following a standard pRF analysis, as described elsewhere (Dumoulin and Wandell, 2008; Harvey and Dumoulin, 2011; Zuiderbaan et al., 2012). For each stimulus condition, we independently estimated the pRF properties using a canonical HRF model (Friston et al., 1998; Glover, 1999; Worsley et al., 2002). These models did not take the speed of motion into account in pRF estimation, only the luminance contrast at each point in the stimulus image, which was the same in all stimulus conditions. Any recording site where the pRF models did not explain at least $10 \%$ of the variance in the time series recorded in every condition was excluded from further analysis.

Next we estimated the HRF parameters that best describe the data of the whole acquired fMRI volume (Harvey and Dumoulin, 2011) for each stimulus condition. For each subject, we then averaged HRF parameters determined from each condition's data and used the resulting HRF parameters to re-estimate $\mathrm{pRF}$ model parameters. This procedure improved the goodness of fit and ensured the same HRF for all conditions and cortical locations. Very similar results were obtained by fitting the data using a canonical HRF (Friston et al., 1998; Glover, 1999; Worsley et al., 2002), again analogous to results we have obtained before (Harvey and Dumoulin, 2011; Harvey et al., 2013).

We identified visual field map borders based on eccentricity and reversals in polar angle of visual field position preference (Sereno et al., 1995) and identified particular visual field maps with reference to previous studies (Smith et al., 2001; Larsson and Heeger, 2006; Swisher et al., 2007; Wandell et al., 2007; Amano et al., 2009). These visual field maps form our regions of interest (ROIs).

\section{Experimental rationale}

Stimulus motion changes the perceived positions of stimuli, separating their perceived positions from their presented positions. We hypothesize that stimulus positions in the neural visual field representation are also affected by motion. We aim to measure the direction and size of these effects in different visual field maps using pRF modeling, revealing where in the visual processing hierarchy they arise.

If stimulus motion continues in the same direction for prolonged periods, this produces perceptual motion aftereffects that are evident in fMRI responses (He et al., 1998; Culham et al., 1999; Huk et al., 2001). Such effects would complicate interpretation of measured responses.

PRF mapping stimuli use bar apertures that gradually step across the visual field. To avoid adaptation effects, we reversed the direction of motion half way through each bar's presentation. Perceptually, the bar appears to move first in one direction then the other.

However, interpretation of the resulting effects on pRF properties is not straightforward (Fig. 2). If pRF positions are displaced in one direction then the other by the same amount, this should increase the estimated pRF size rather than move the pRF preferred position. Consequently, apparent changes in pRF size may reflect actual changes in pRF size, or sequential changes in pRF position only.

If these sequential changes in pRF position are of different sizes, this imbalance should also change in pRF position estimates. For example, if pRF displacements away from fixation are larger than pRF displacements towards fixation, pRF eccentricity estimates should increase.

We examined responses to two different directions of motion: in the direction that the pRF mapping bar steps across the visual field and orthogonal to this direction (Fig. 1A). Responses to any single bar pass do not tell us which part of the bar is stimulating the pRF. Therefore, a single bar pass only informs the model about the pRFs position and size in the direction of bar movement (Dumoulin et al., 2014). The direction of the bar steps is therefore the direction of effective pRF measurement. 


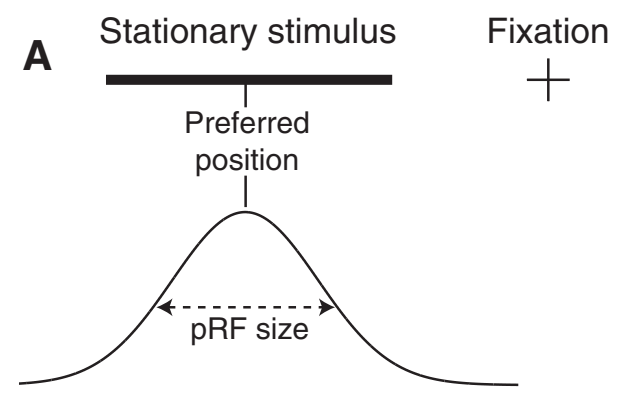

Separate pRF displacements

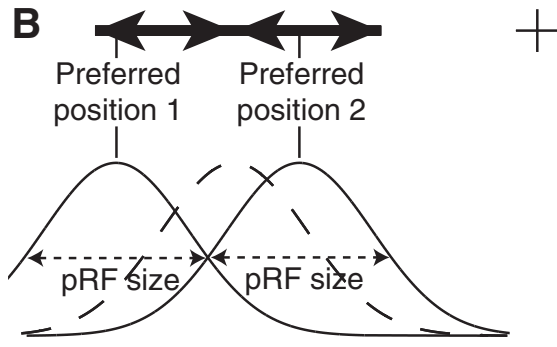

Summed pRF displacements

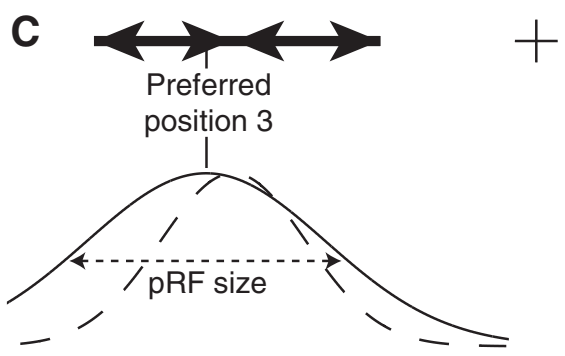

Fig. 2. Possible mechanisms of pRF size and position changes. (A) PRF of a recording site whose preferred position for stationary stimuli corresponds to the middle of the stimulus, shown as dashed lines in other panels. (B) Sequential presentation of two opposite motion directions (towards and away from fixation) may produce large changes in pRF preferred positions away from fixation and smaller changes towards fixation, without necessarily affecting pRF size. Note that our experiments use translational motions, not expanding and contracting motions. However, all motion directions move towards or away from fixation to some extent. (C) These two sequential changes cannot be distinguished by the temporal resolution of fMRI. Their combined effects may increase pRF size estimates, although neither stimulus direction necessarily affects pRF size. If pRF preferred position changes away from fixation are larger than changes towards fixation, this would increase pRF eccentricity estimates in the combined response.

Only effects of motion in the direction of pRF measurement will affect measured pRF properties: effects orthogonal to the direction of pRF measurement should remain within the stimulated area, and so should not affect the responses seen.

As very different neuronal populations process moving and stationary stimuli, we do not compare pRFs recorded with stationary stimuli. We only examined changes in pRF properties between stimuli with different motion speeds. Therefore, we could not characterize speed independent effects that would be the same for all motion speeds.

\section{Comparisons between stimulus conditions}

To compare the pRF properties between different stimulus conditions, we grouped data from all subjects for recording sites in the same visual field map. For every visual field map, we used a paired samples procedure to remove variance in pRF parameters between recording sites and subjects. For every recording site, we first determined the pRF size and eccentricity in every condition relative to the mean across all conditions for that site. We used the resulting relative pRF size or eccentricity as inputs to a general linear model (GLM). The GLM determined the contribution of the speed-dependent slope term to pRF size and eccentricity estimates. These GLMs also fit a common baseline pRF size across all conditions, though this term was not analyzed. To visualize the differences between conditions, we first determined the mean and standard error of the relative pRF size and eccentricity for every condition. We then determined the mean pRF size and eccentricity across all conditions and recording sites within the visual field map. We added this to each relative parameter estimate to give the values shown in Fig. 5A \& B. The slope (beta) term of each GLM is shown in Fig. 5C \& D.

To compare the effects of motion in the direction of pRF measurement to those in the orthogonal direction (Fig. 1A), we examined the difference between two speeds $\left(2.5^{\circ} / \mathrm{s}\right.$ and $\left.5^{\circ} / \mathrm{s}\right)$ for each direction. For each motion direction and visual field map we compared pRF size and eccentricity estimates obtained for the same recording sites at different speeds using paired samples $t$-tests. Again, these paired tests characterize differences between conditions and remove variance between recording sites and subjects. Because different grating orientations were used for motion in these two directions, different directions were not compared to each other (Dumoulin et al., 2014). All comparisons were corrected for upsampling during interpolation to anatomical space.

\section{Psychophysics}

Following stimuli used for fMRI pRF mapping, all psychophysics stimuli were generated in Matlab (Mathworks, Natick, MA, USA) using the PsychToolbox (Brainard, 1997; Pelli, 1997). They were presented on a $40.0 \times 30.0 \mathrm{~cm}$ CRT display that had been gamma-corrected using the output of a PR650 colorimeter (Photo Research Inc., Chatsworth, CA, USA). The display was $154 \mathrm{~cm}$ from the subjects' eyes, giving a visual angle of $11^{\circ}$ for the vertical dimension of the display. Display resolution was $1024 \times 768$ pixels.

To examine the perceptual effects of our fMRI stimuli, our psychophysics experiments quantified the perceived position of one of the stimulus bar-aperture positions that would be used for fMRI visual field mapping (Figs. 3A \& 1A). As in our fMRI stimuli, the contrast of the bar-aperture faded at its edges following a $1^{\circ}$ wide raised cosine, and including these edges, the bar-aperture was $2^{\circ}$ wide. The center of this bar-aperture was on average at $2.75^{\circ}$ eccentricity, the middle of the eccentricity range shown in the fMRI experiment. The baraperture was horizontal, above the fixation point and contained a horizontally oriented grating. We showed this bar in two intervals, whose positions the subjects compared. Each interval was $800 \mathrm{~ms}$ long including $50 \mathrm{~ms}$ fades of linearly increasing and decreasing contrast at the start and end (respectively) of each interval. These fading onsets and offsets avoided strong position cues during the appearance and disappearance of the stimulus (Fig. 3B). There was an interval of $500 \mathrm{~ms}$ between the end of the first interval's offset and the start of the second interval's onset.

We systematically varied the vertical distance between the two baraperture presentations. Each bar-aperture presentation contained either upwards motion, downwards motion, or no motion. The subjects judged whether the second bar-aperture presentation was higher or lower than the first. To avoid subjects comparing stimulus positions to fixed reference positions, each trial was randomly jittered in vertical position, following a Gaussian distribution with a standard deviation of $0.1^{\circ}$. In every interval, the grating started at a random phase. All comparisons were either between presentations of the same speed or with one interval remaining stationary. Comparisons of single motion directions to stationary stimuli allowed us to separate effects of the two opposite motion directions, which were shown together during pRF mapping.

All stimulus conditions were shown in one randomly ordered mixed block, which contained all motion speeds used in pRF mapping experiments $\left(1.25^{\circ} / \mathrm{s}, 5^{\circ} / \mathrm{s}, 3.75^{\circ} / \mathrm{s}\right.$ and $\left.5^{\circ} / \mathrm{s}\right)$, all vertical offsets between bar- 


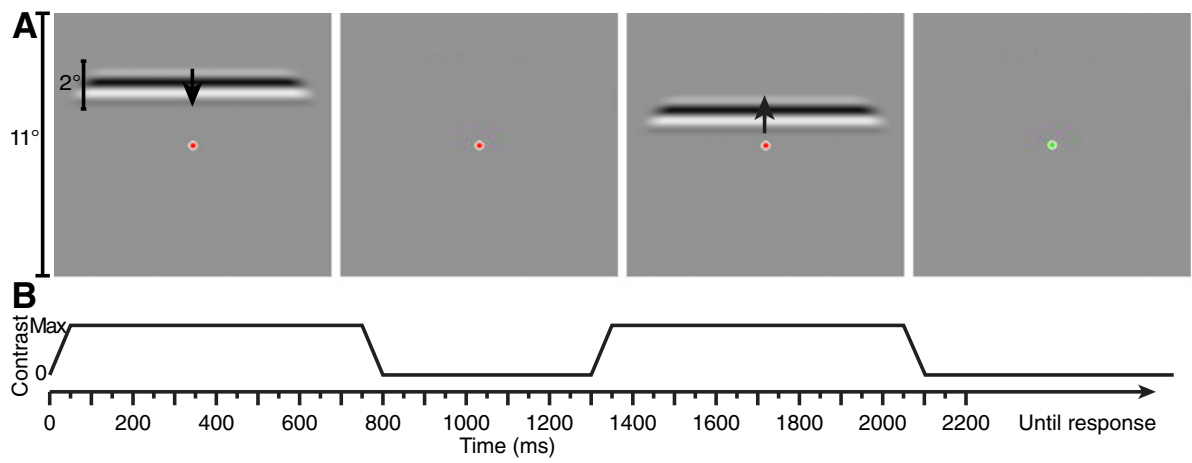

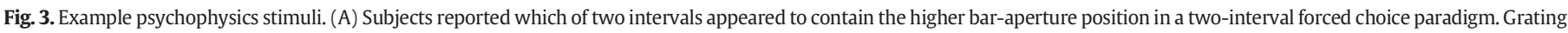

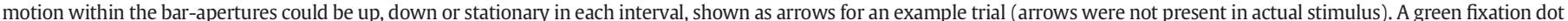

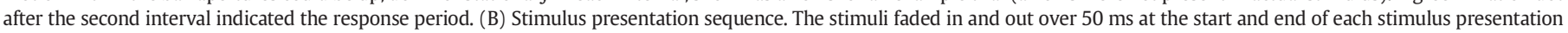
respectively. Each stimulus was presented at $77 \%$ or $5 \%$ Michelson contrast for 700 ms throughout an entire testing session. Presentations were separated by an interval of 500 ms.

aperture positions (the second bar-aperture at the same location as the first, or $0.05^{\circ}, 0.1^{\circ}, 0.2^{\circ}, 0.4^{\circ}$, or $0.8^{\circ}$ above or below the first) and all eight combinations of motion directions (up then down, down then up, down then down, up then up, up then stationary, stationary then up, down then stationary, and stationary then down). This block therefore contained 352 trials, taking about 15 min to complete one block. Each subject performed eight repeated blocks, each ordered differently. We first performed this experiment at high contract (as in the fMRI experiment). We then repeated the psychophysics experiment (but not the fMRI experiment) at 5\% Michelson contrast, which increased perceptual effect sizes and so allowed more accurate quantification of perceptual effects.

To examine perceptual effects of pRF mapping stimuli with motion orthogonal to the direction of pRF measurement, we also measured effects on perceived position when the motion direction was orthogonal to the bar-aperture displacement. As for pRF mapping stimuli, we used an orthogonally oriented grating (Fig. 1A).

\section{Psychophysics analysis}

To avoid effects of response bias, we first combined trials based on the relative motion direction of the two intervals. This resulted in four relative motion direction conditions: same direction, opposite direction, up versus stationary and down versus stationary. To allow comparisons between up versus stationary and down versus stationary conditions, responses to the latter condition were reversed. For each of these direction conditions and each motion speed, we used the psignifit toolbox (Frund et al., 2011) to fit a cumulative Gaussian psychometric function for the likelihood of perceiving the second bar-aperture as higher, against the difference between bar-apertures positions (Fig. 9A). This determined the point of subjective equality (PSE, where both intervals appeared to contain bar-apertures at the same location) and its $95 \%$ confidence intervals by bootstrapping.

This analysis was done for responses from every individual subject, and also for grouped response from all subjects. For grouped data, responses from all trials were grouped together before fitting psychometric functions and confidence intervals. We analyzed the effect of motion speed on each direction condition using a GLM to fit speed-dependent effects (a slope term describing the change in PSE with speed) and speedindependent effects (an intercept term). These models provide separate $t$-statistics for speed-dependent and speed-independent effects.

\section{Results}

\section{Motion speed affects fMRI responses}

We characterized fMRI BOLD responses to bar-apertures containing moving gratings and crossing the visual display in several directions
(Fig. 1B). Faster motion speeds typically increased the duration of the BOLD response to each pass of the bar-aperture (Fig. 4). Consequently, faster motions typically produce neural responses over a larger area of the visual field.

\section{Effects of motion speed are captured by changes in $p R F$ size and position}

We summarized the responses of each recording site at each speed using pRF models that describe the size and position of the part of the visual field to which each recording site responds. We first use GLMs to examine how pRF size and pRF preferred position eccentricity ( $\mathrm{pRF}$ eccentricity) are affected by motion speed.

PRF sizes increased up the visual hierarchy. Within all visual field maps examined except hV4, pRF sizes increased significantly with increasing motion speed (Fig. 5A, Table 1). The magnitude of this effect increased up the visual processing hierarchy (Fig. 5A \& C). In later visual field maps pRF sizes can change by over $1.5^{\circ}$ between fast and slow speeds.

Despite this speed-dependent pRF size increase, the slowest speed tested $\left(1.25^{\circ} / \mathrm{s}\right)$ yielded larger pRF sizes than some faster speeds. All statistics described above include the $1.25^{\circ}$ /s condition in the GLM. If we remove data acquired with $1.25^{\circ} / \mathrm{s}$ from the GLM, the speed-dependent pRF size increase becomes significant in all visual field maps (all $p \leq 0.0004$ ). Furthermore, removing any speed condition from analysis does not affect the pattern of significant effects seen. Therefore, model fits are not driven by any single speed condition.

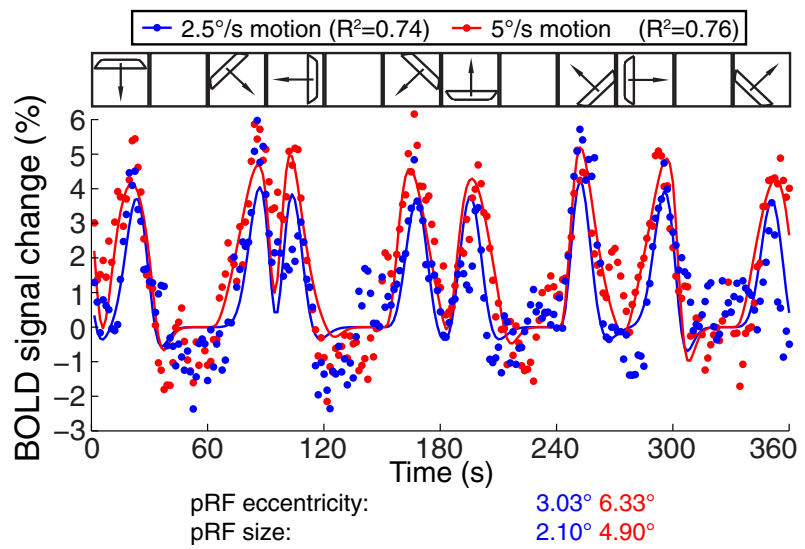

Fig. 4. FMRI time series for the same recording site in V3A when stimuli contain motion in the direction of pRF measurement at $2.5 \% \mathrm{~s}$ (blue) and $5 \% \mathrm{~s}$ (red). Motion at $5 \% \mathrm{~s}$ produced longer lasting responses, covering more of the visual field. Points show the recorded data, lines show the predicted fMRI time courses of pRF models fits to this data. 
A

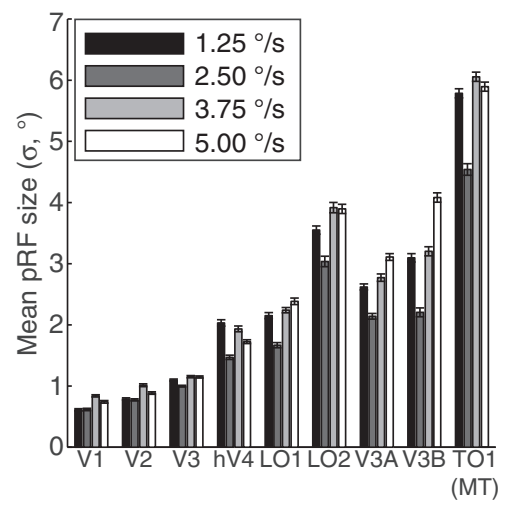

B
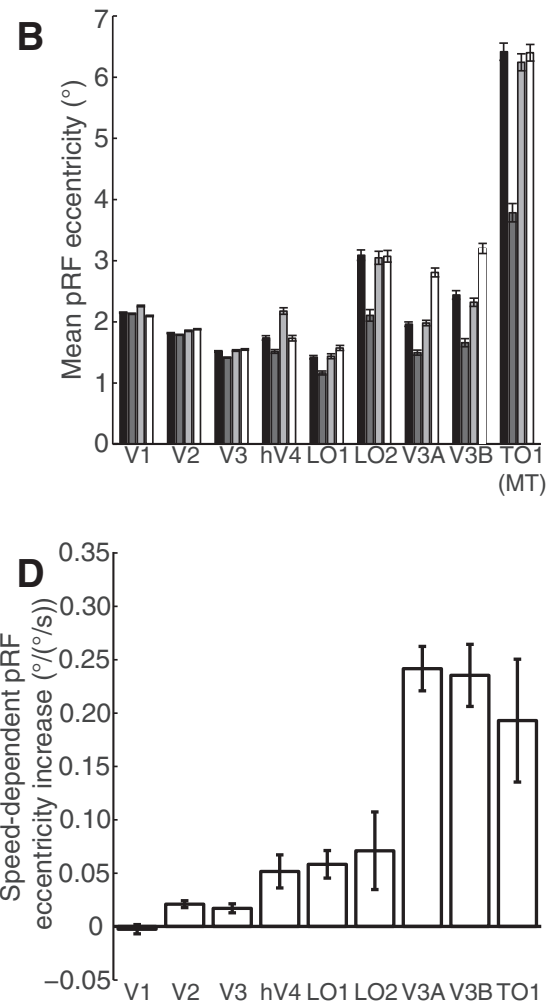

(MT)
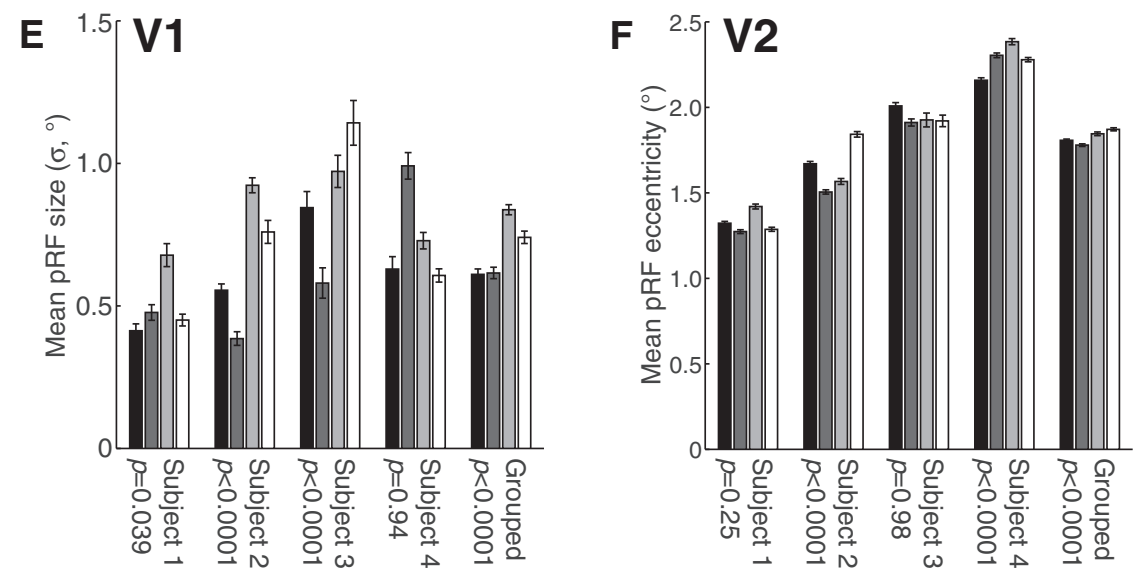

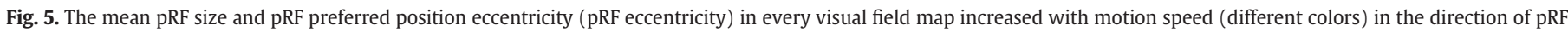

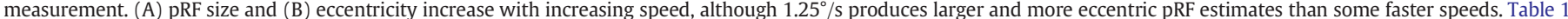

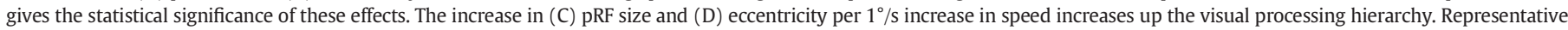

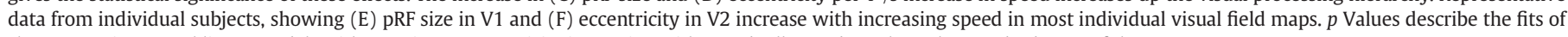
slope terms in general linear models with pRF size or eccentricity increasing with speed. All error bars show the standard error of the mean.

PRF eccentricity also increased significantly with increasing speed in all visual field maps except V1 (Fig. 5B \& D, Table 1). Again, this effect was larger for the slowest speed tested than for some faster speeds. Again, removing any speed condition from analysis does not affect the overall pattern of significant effects seen.

These effects in grouped data were also consistently found in individual subjects. Excluding hV4 (where no effects of speed on pRF size were found), pRF sizes increased significantly with motion speed in $66 \%$ of individual subject's visual field maps examined. Representative results from V1 are shown in Fig. 5E. Similarly, excluding V1 (where no effects of speed on pRF eccentricity were found) pRF eccentricity increased significantly with motion speed in 56\% of individual visual field maps examined. Representative results from V2 are shown in Fig. 5F.
Comparisons so far have used GLMs to examine differences in pRF size and eccentricity. A simpler analysis uses paired $t$-tests to compare pRF sizes and eccentricities measured from the same recording sites in the two most different conditions: $2.5^{\circ} / \mathrm{s}$ and $5^{\circ} / \mathrm{s}$ (Table 1 ). Like the GLM analysis, this simpler analysis demonstrates that pRF sizes increase significantly with increasing speed in all visual field maps, and pRF eccentricities increase significantly with increasing speed in all visual field maps except V1. Analyses from this point on will only compare these two speeds.

\section{Correlations between $p R F$ size changes, eccentricity changes, and pRF size}

Increases in pRF size and eccentricity become larger up the visual hierarchy, as does pRF size (Fig. 6). Each visual field map's increase in 
Table 1

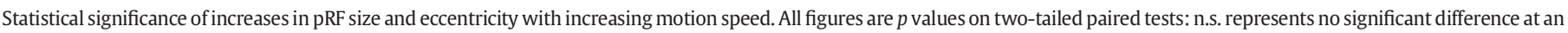
alpha level of 0.05 . Negative figures represent decreases in pRF size or eccentricity with increasing motion speed.

\begin{tabular}{|c|c|c|c|c|c|c|}
\hline \multirow{2}{*}{$\begin{array}{l}\text { Visual field } \\
\text { map }\end{array}$} & \multicolumn{4}{|c|}{ Motion in direction of pRF measurement } & \multicolumn{2}{|c|}{ Orthogonal motion direction } \\
\hline & $\begin{array}{l}\text { pRF size increase } \\
\text { (GLM) }\end{array}$ & $\begin{array}{l}\text { Eccentricity increase } \\
\text { (GLM) }\end{array}$ & $\begin{array}{l}\text { pRF size increase } \\
\left(5^{\circ} / \mathrm{s}-2.5^{\circ} / \mathrm{s}\right)\end{array}$ & $\begin{array}{l}\text { Eccentricity increase } \\
\left(5^{\circ} / \mathrm{s}-2.5^{\circ} / \mathrm{s}\right)\end{array}$ & $\begin{array}{l}\text { pRF size increase } \\
\left(5^{\circ} / \mathrm{s}-2.5^{\circ} / \mathrm{s}\right)\end{array}$ & $\begin{array}{l}\text { Eccentricity increase } \\
\left(5^{\circ} / \mathrm{s}-2.5^{\circ} / \mathrm{s}\right)\end{array}$ \\
\hline V1 & $<0.0001$ & n.s. & $<0.0001$ & n.s. & n.s. & n.s. \\
\hline V2 & $<0.0001$ & $<0.0001$ & $<0.0001$ & $<0.0001$ & n.s. & n.s. \\
\hline V3 & 0.003 & $<0.0001$ & $<0.0001$ & $<0.0001$ & n.s. & n.s. \\
\hline hV4 & n.s. & 0.0005 & $<0.0001$ & $<0.0001$ & $-<0.0001$ & -0.023 \\
\hline L01 & $<0.0001$ & $<0.0001$ & $<0.0001$ & $<0.0001$ & -0.0003 & n.s. \\
\hline LO2 & $<0.0001$ & 0.026 & $<0.0001$ & 0.0005 & n.s. & 0.003 \\
\hline V3A & $<0.0001$ & $<0.0001$ & $<0.0001$ & $<0.0001$ & $-<0.0001$ & n.s. \\
\hline V3B & $<0.0001$ & $<0.0001$ & $<0.0001$ & $<0.0001$ & n.s. & n.s. \\
\hline T01 (MT) & $<0.0001$ & 0.0004 & 0.036 & 0.0003 & n.s. & n.s. \\
\hline
\end{tabular}

pRF size is correlated with its mean pRF size across both speed conditions $(r=0.86, p=0.003)$, as is its increase in eccentricity $(r=0.96$, $p<0.0001)$. Increases in pRF size and eccentricity are also correlated $(r=0.79, p=0.01)$. Furthermore, within each map, increases in each recording site's pRF size are correlated with its mean pRF size across both speed conditions (all $r>0.095, p<0.002$ ). With the exception of V1 (were no significant eccentricity change was found) and V2, increases in each recording site's eccentricity are also correlated with its mean $\mathrm{pRF}$ size across both speed conditions (all $\mathrm{r}>0.16, p<0.0001$ ) and its increase in pRF size (all $r>0.19, p<0.0001$ ). The slope of this relationship is similar across visual field maps suggesting pRF effects are determined more by a recording site's pRF size than by its visual field map: recording sites with similar pRF sizes in different visual field maps show similar effect sizes (Fig. 6).

\section{$P R F$ effects are specific to motion in the direction of $p R F$ measurement}

So far, we have tested grating motion in the direction of pRF measurement (Fig. 1A). Based on our rationale (see 'Experimental rationale' section and Dumoulin et al, 2014), motion orthogonal to the direction of pRF measurement, should not affect pRF estimates. On the other hand, another interpretation would be that pRF size differences could result from neurons with larger receptive fields processing faster speeds or higher temporal frequencies, or faster speeds producing larger response amplitudes that might be fit as larger pRFs. None of these explanations are consistent with the directional effects on pRF eccentricity. To exclude these explanations for pRF size effects and verify our predictions, we examined speed-dependent pRF size and eccentricity differences when the grating moved orthogonal to the direction of pRF measurement (Fig. 1A) in three of our fMRI subjects (Figs. 7 \& 8). To make the grating move orthogonal to the direction of pRF measurement, we rotated its orientation by $90^{\circ}$ (Fig. $1 \mathrm{~A}$ ).
Unlike motion in the direction of pRF measurement, orthogonal motion did not consistently increase pRF sizes (Figs. 7A \& 8A) or eccentricity (Figs. 7B \& 8B) at higher speeds. Indeed, several visual field maps show significantly smaller pRF sizes at higher speeds for orthogonal motion, suggesting a speed-dependent elongation of the $\mathrm{pRF}$ in the direction of motion coupled with a thinning of the pRF in the orthogonal direction. However, this effect is less consistent between visual field maps than the effect of motion in the direction of pRF measurement. We were not able to model effects of direction on pRF shape directly here: motion direction changed throughout each scan as the direction of pRF measurement changed. A different experimental design would be needed to determine effects of motion on pRF shape.

As pRF size and position changes in each visual field map are well correlated to pRF sizes, we ask how these changes progress through the hierarchy when expressed as a proportion of pRF size (Fig. 8C \& D). This analysis reveals that changes in pRF size are approximately proportional to $\mathrm{pRF}$ size. Changes in $\mathrm{pRF}$ eccentricity are absent from V1 but are approximately proportional to pRF size across other visual field maps.

Finally, we test whether our ability to detect pRF changes depends on the strength of responses to our stimuli. For example, small responses at the edge of the pRF might not distinguishable from baseline where signal strength is low. Furthermore, improved signal to noise ratio might improve our ability to detect changes, making changes appear larger. While we don't believe either of these effects introduce systematic biases in pRF estimates (but rather affect non-systematic measurement noise), Fig. 9 examines how the goodness of pRF model fits differs with speed in different visual field maps, and in both motion directions, for comparison with Fig. 8. While goodness of fit differs with speed and direction, there is no clear relationship between these changes in and the changes in pRF properties shown in Fig. 8: increased pRF size or eccentricity can occur with increased or decreased goodness of
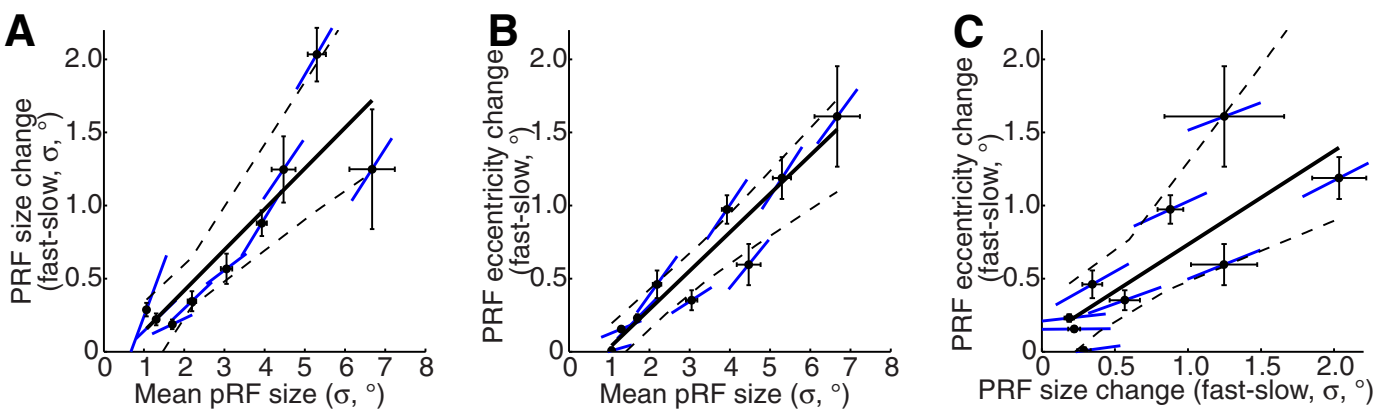

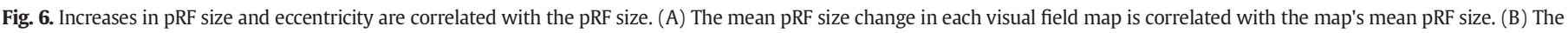

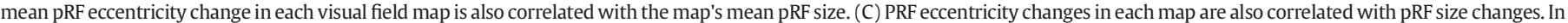

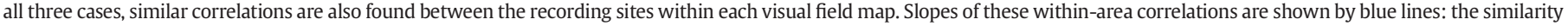

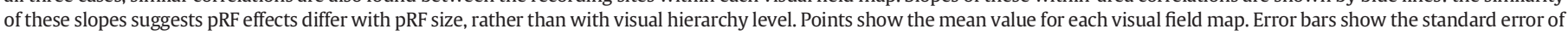
the mean. Black slopes show the best fitting slope of these means, dashed lines show the $95 \%$ confidence intervals of this slope, determined by bootstrapping. 

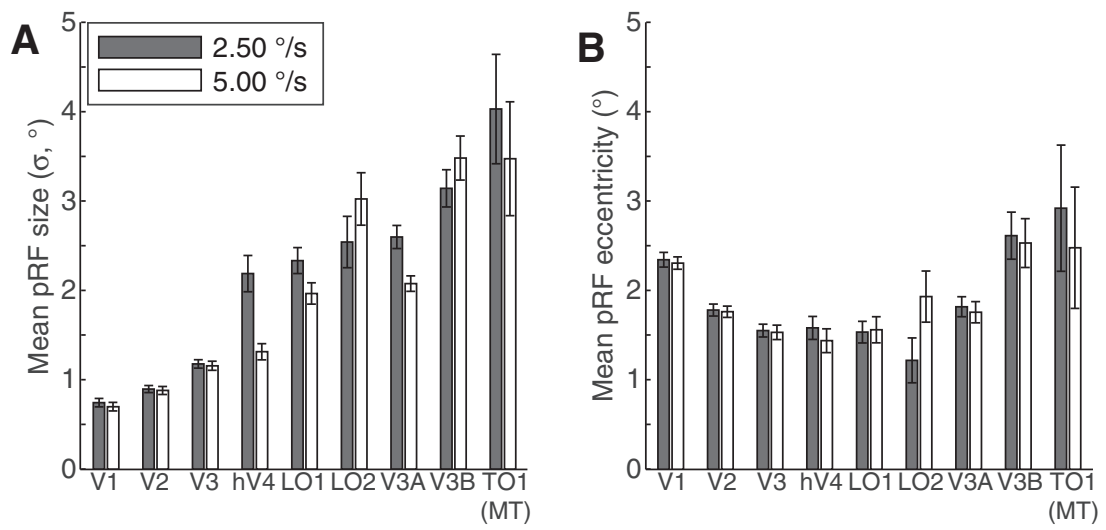

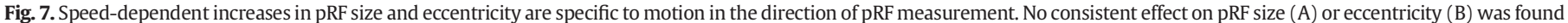
when motion was orthogonal to the direction of pRF measurement.

model fits. Therefore, simple changes in signal to noise ratio cannot explain our results.

\section{Perceptual displacement is affected by motion speed, direction and contrast}

Grating motion within the stationary bar-apertures used in pRF mapping moved their perceived position in the direction of grating motion. We quantified this perceived displacement as the point of subjective equality (PSE) where physically separated gratings appeared to be in the same place (Fig. 10A). We characterized the effect of speed on the extent of perceptual displacement using a general linear model (GLM). The GLM fit both speed-dependent (slope) and speedindependent (intercept) effects. Unless otherwise stated, $p$ values are for two-sided comparisons from these GLMs.

High contrast stimuli produced small perceptual displacements of bar-aperture position (Fig. 10B). When subjects compared a bar-
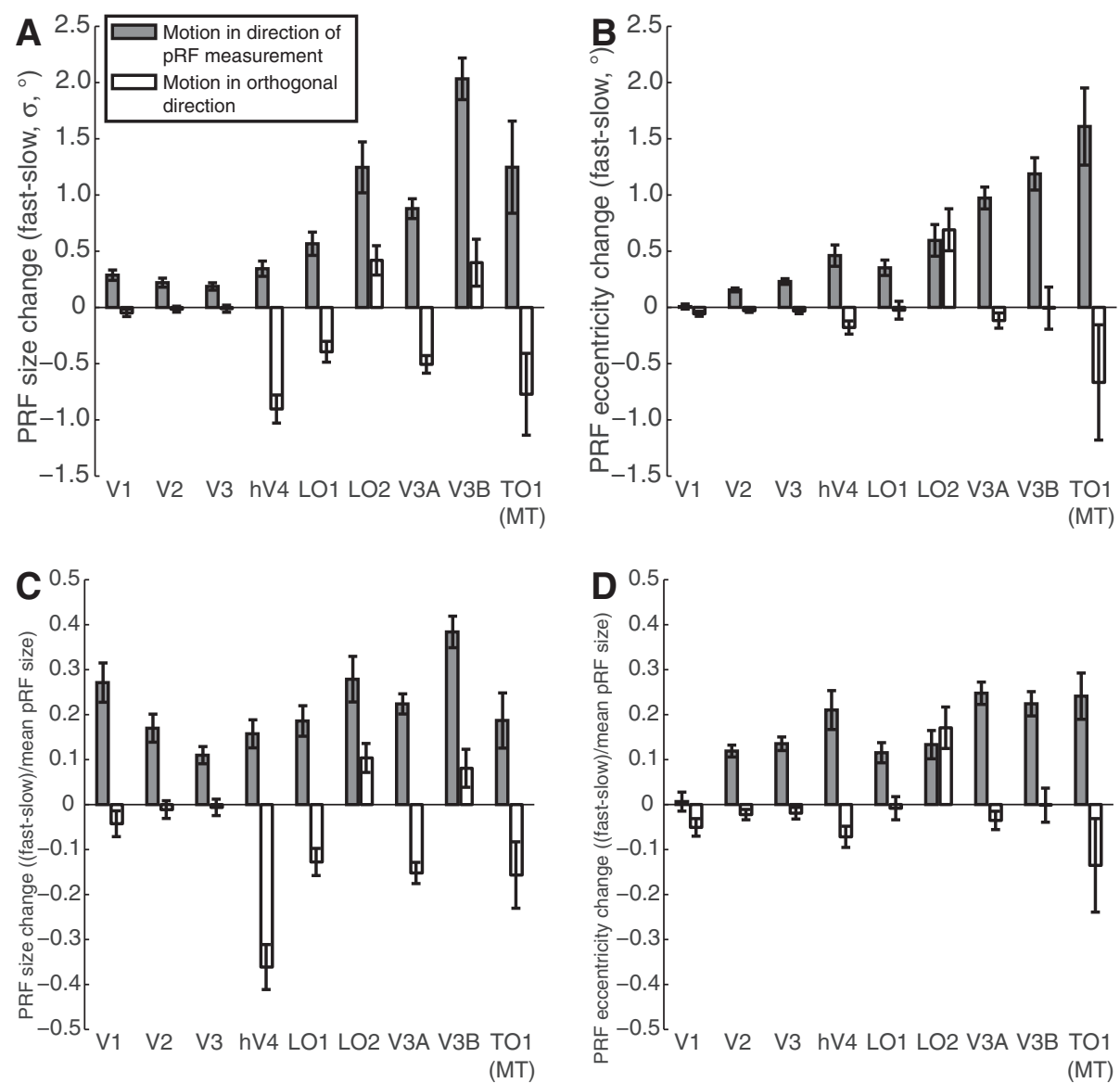

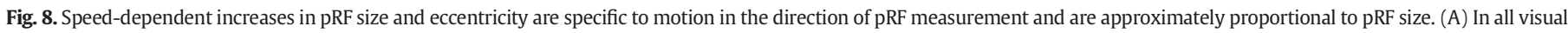

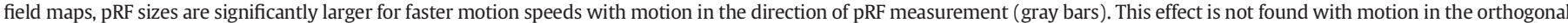

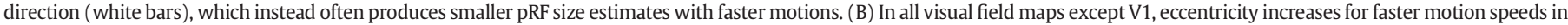

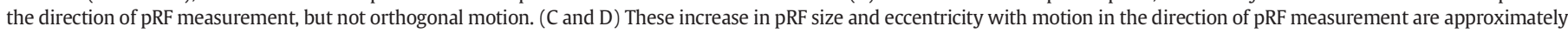
proportional to pRF size. However, changes in pRF eccentricity are absent in V1. All error bars show the standard error of the mean. 


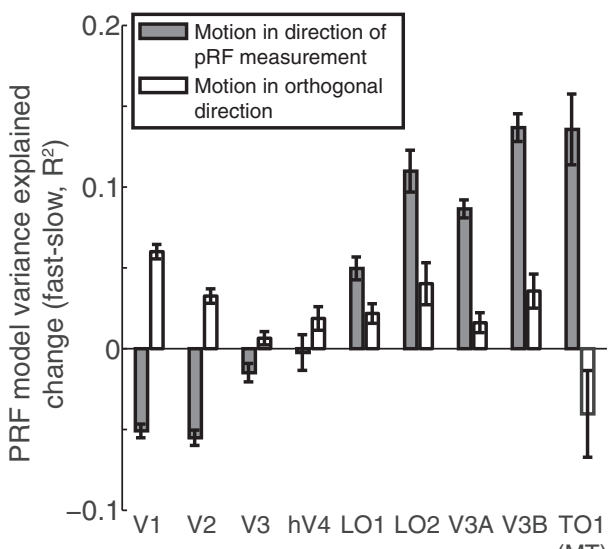

(MT)

Fig. 9. Speed- and direction-dependent changes in the goodness of pRF model fits. While goodness of fit differs between motion speeds in both directions, these changes show little relationship to the changes in pRF parameters shown in Fig. 8. So simple changes in signal to noise ratio alone cannot explain our results.

aperture containing a moving grating to a bar-aperture containing a stationary grating, the perceived bar-aperture position was displaced in the direction of motion. This effect was found for gratings moving either towards $(p=0.01)$ or away $(p=0.01)$ from fixation. The perceived displacement was larger for gratings moving towards fixation (down) than gratings moving away (up) $(p=0.03)$. The displacement did not change significantly with motion speed in either comparison. When two gratings moved in the same direction, these appeared to be in the same position. When the two gratings moved in opposite directions (as in fMRI stimuli), perceived displacement was approximately the sum of comparisons of each moving grating with a stationary grating. This displacement increased slightly with speed, reaching significance on one-sided tests only ( $p=0.03$, one-sided), but showed a more significant speed-independent displacement $(p=0.002)$. The speed dependent effect reached significance in one individual subject only. Other effects were similar in every subject, though two subjects did not perceive gratings moving away from fixation as significantly displaced. Overall, we find some effects of motion direction on perceived position here, but the perceptual displacement does not increase strongly with increasing speed, so it does not seem to closely follow the effects of motion speed on pRF size and eccentricity.

As perceptual effects at high contrast were weak and speeddependent perceptual effects were only marginally significant, we reduced the contrast of the stimulus to target the direction selective neurons more specifically. This produced far larger perceptual displacements (Fig. 10C). Bar-apertures containing gratings moving away from fixation were significantly displaced $(p=0.0002)$ but this displacement still did not increase significantly with motion speed. Gratings moving towards fixation were increasingly displaced with increasing speed ( $p=0.009$ ), following an approximately linear function. This function also had a significant speed-independent component $(p=0.003)$. As with high contrast stimuli, displacement was larger for motion away from fixation than motion towards fixation $(p=0.043$, two-sided paired $t$-test). Again, the sum of these two comparisons with stationary gratings approximated the comparison between two gratings moving in opposite directions. As one of these presentations contained a grating moving towards fixation, displacement increased significantly with speed $(p=0.01)$ but also had a significant speedindependent component $(p=0.002)$. When both gratings moved in the same direction, they were not significantly displaced relative to each other. We found similar results in every subject. However, one of our four subjects did not show significant speed-dependent effects for gratings moving towards fixation ( $p=0.12$, one sided) or gratings moving in opposite directions ( $p=0.28$, one sided). In two subjects, displacement of gratings moving away from fixation did not reach significance, though trends were present here $(p=0.066$ and $p=0.0501$, one sided). Overall, when we reduce stimulus contrast we find much larger perceptual displacements. These larger displacements reveal significant speed-dependent effects for motion towards fixation. These may have been present for high contrast stimuli, but were too small to reach significance. Nevertheless, speed-independent effects dominated all perceptual displacements at both contrasts.

We also confirm, in one subject, that motion towards fixation is displaced more than motion away from fixation when bar apertures are presented below fixation. So this direction asymmetry does not seem to reflect different larger perceptual displacements for downwards than upwards motion.

We also tested whether horizontally moving gratings produced vertical perceptual displacements. Here the PSE was not significantly different from zero for any combination of motion directions at any speed. So grating motion does not affect perceived position in the orthogonal direction.

\section{Discussion}

In this study, we asked how visuo-spatial response preferences are affected by motion in visual stimuli, where in the visual processing hierarchy these effects occur, and how they progress throughout the hierarchy. Our results demonstrate changes in pRF properties with stimulus motion speed in the direction of pRF measurement. In all visual field maps, pRF sizes increased with motion speed. Except in V1, pRF
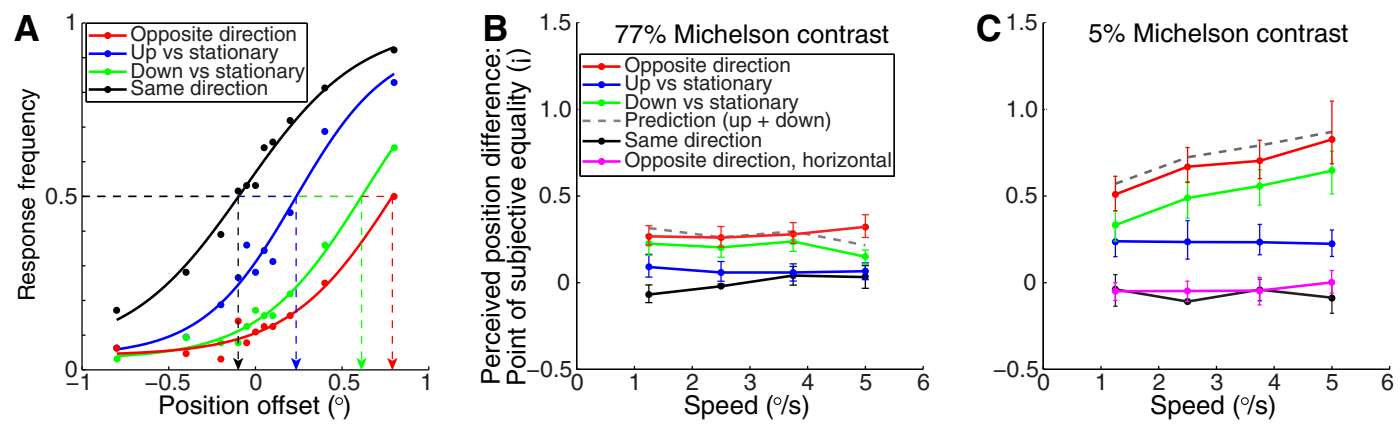

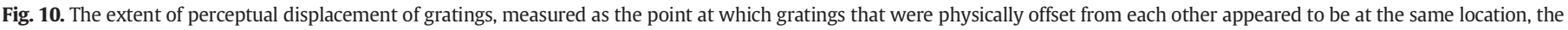

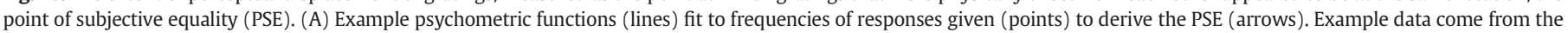

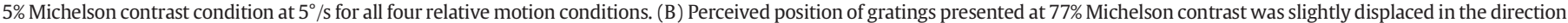

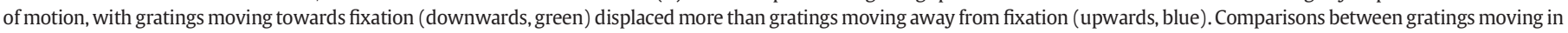

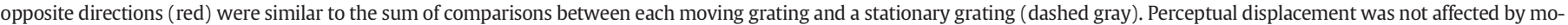

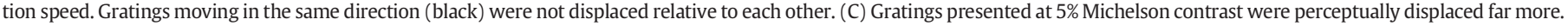

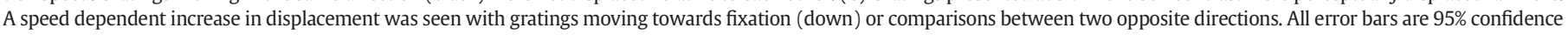
intervals determined by bootstrapping. 
eccentricity also increases. These pRF effects become larger up the hierarchy, approximately in proportion to $\mathrm{pRF}$ size. Within each visual field map, pRF effects are also approximately proportional to pRF size. The slope of this proportionality is similar in each visual field map, suggesting similar transformations of each visual field map's representation of visual space. Similar effects are not induced by motion orthogonal to the direction of pRF measurement. We also examine perceptual displacements of these stimuli. Consistent with our interpretation of pRF eccentricity changes (described below), perceptual displacements are larger for motion towards fixation. However, the perceptual displacement is largely independent of motion speed: speed-dependent perceptual displacements are only evident when stimulus contrast is reduced, which increases the extent of perceptual displacements. As such, perceptual displacements do not closely reflect changes in visuo-spatial response preferences.

\section{Experimental paradigm and interpretation}

These experiments aimed to show whether pRFs were affected by motion speed and direction, and to compare the size of these effects in different visual field maps. However, it was not possible to design an experiment that straightforwardly quantified pRF size and position changes independently without adaptation effects that would complicate the interpretation of any results, as discussed below. In our design, pRF mapping stimuli sequentially showed two opposite motion directions, both of which probably affect responses. Together with fMRI's limited temporal resolution, this prevents us from distinguishing pRF size and position changes (Fig. 2). The reported pRF size increases may result from sequential displacements of receptive field preferred positions in two opposite directions, which may or may not be accompanied by changes in the underlying receptive field sizes (see Section Experimental rationale).

Further experiments might isolate pRF position changes between stimulus conditions that each contain single motion direction throughout a scan run. However, motion adaptation effects from prolonged unidirectional motion presentation would complicate interpretation of any results obtained. First, any observed pRF position displacement could result from the motion direction in the stimulus or from the opposite motion direction in a motion aftereffect. Therefore, as in our experimental design, any observed pRF displacement could result from either of two opposite motion directions and would probably result from a combination of both. Second, motion adaptation aftereffects keep fMRI response amplitudes above baseline after the stimulus has disappeared (He et al., 1998; Culham et al., 1999; Huk et al., 2001). With a pRF mapping stimulus that steps across the visual field, this would broaden the range of stimulus positions where responses are observed, and so increase pRF size estimates. Therefore, as in our experimental design, any observed increase in pRF sizes could result from the details of the experimental design.

For future experiments, we also note that motion towards and away from fixation seem to have quite different effects. So translational motion directions may have different effects at different polar angles. If both neural and perceptual adaptation effects could be sufficiently avoided, comparing pRFs measured separately with expanding and contracting motions at various speeds to those measured with stationary flicker may be optimal for disentangling the various effects we find.

PRF properties change throughout the hierarchy, but we cannot separate the contributions of different visual field maps to perception. Given our experimental design, changes in pRF preferred position may appear as changes in PRF size, and we cannot determine the size of pRF displacements produced by single motion directions. Effects on pRF eccentricity instead reflect differences between effects of motion towards and away from fixation. Therefore, we cannot compare the extent of perceptual displacement to the extent of pRF displacement in each visual field map, which might link perceptual displacements to particular visual processing levels. We also used different subjects to measure effects on pRFs and perception, and only four subjects in each. Consequently, we cannot quantitatively compare the extents of pRF displacement and perceptual displacement, either at the group level or the inter-individual level.

Previous studies have compared the extent of human perceptual displacement to the extent of receptive field effects in cat V1 (Fu et al., 2004) or macaque V4 (Sundberg et al., 2006). Because pRF size and eccentricity are affected to different extents in different visual field maps, we avoid such comparisons. It seems unlikely that perceived position reflects neural activity in a single area.

Motion towards and away from fixation are perceptually displaced to different extents, and effects on pRF eccentricity suggest that these directions also affect pRFs preferred positions to different extents. This asymmetry may arise from center-biased visual field map organization. As cortical magnification factors decrease with eccentricity, the same cortical distance covers more visual space moving away from fixation than towards fixation. When early visual field maps are sampled by later areas (Motter, 2009; Harvey and Dumoulin, 2011; Haak et al., 2012) the resulting receptive field extends further away from fixation than towards fixation (Motter, 2009). If cortical sampling limitations underlie speed-dependent changes in pRF eccentricity (as discussed in the section Mechanism of preferred position changes), pRF eccentricity changes would be absent from V1, as seen. Alternatively, effects on pRF eccentricity in V1 may simply be too small for us to detect, although the clearly significant effects on pRF size by V1 do not favor this interpretation.

Perhaps counter-intuitively, we expect neural position preferences and perceived positions to be displaced in opposite directions. For example, when a central visual field pRF is displaced towards the periphery, peripheral stimulation will cause a response that is normally associated with central stimulation. Therefore, according to a labeled-line code, the stimulus will appear more central because the pRF becomes more peripheral.

Our fMRI results do not conclusively demonstrate that pRF preferred positions are displaced against the direction of stimulus motion, because we present two directions of motion in the same interval and cannot distinguish their individual effects on pRF position preferences. We believe that pRF preferred positions are displaced against the direction of motion by reference to previous fMRI and neurophysiological results. FMRI response amplitudes increase in recording sites representing the location where motion appears (Whitney et al., 2003; Liu et al., 2006; Raemaekers et al., 2009; Schellekens et al., 2013; Maloney et al., 2014). The recording sites with increased response amplitudes have pRFs within the stimulus area (Liu et al., 2006). Therefore, larger responses at the location where motion appears will displace the most responsive part of the $\mathrm{pRF}$ towards that location, against the direction of motion. Furthermore, neurophysiological studies show receptive field displacement against the direction of motion (Fu et al., 2004; Sundberg et al., 2006). Consequently, considering our results together with previously reported effects of single motion directions on fMRI activation and receptive field properties, we believe that pRF preferred positions are displaced against the direction of motion.

The perceptual displacements we find do not closely follow changes in pRF properties. Specifically, effects on pRF size and eccentricity are strongly speed-dependent, while psychophysical results (at both high and low contrast) are dominated by speed-independent effects. However, we interpret this difference with caution for two reasons. First, we only compare pRFs measured with moving stimuli, so we cannot characterize speed-independent effects that would be present for all of these stimuli. Second, we cannot conclude that there are no speed-dependent perceptual effects for high contrast stimuli: such effects may be too small to reach significance in our specific experiment. Rather than concluding that speed dependent effects were absent, we reduced stimulus contrast to make all perceptual effects larger. Speeddependent effects are quite clear with these larger perceptual displacements, though again speed-independent effects are much larger. So we 
do not conclusively demonstrate that pRFs do not exhibit speedindependent effects or that position perception in high-contrast does not exhibit speed-dependent effects.

\section{Relationships to previous literature}

Single neuron recordings also show effects on receptive field properties (Fu et al., 2004; Sundberg et al., 2006). PRFs are larger than single neuron receptive fields (Smith et al., 2001; Dumoulin and Wandell, 2008; Harvey and Dumoulin, 2011), but position and size changes may be similar for pRFs and single neuron receptive fields. Therefore, these changes may be larger proportions of single neuron receptive field sizes than pRF sizes.

Eye movements affect pRF estimates (Levin et al., 2010; Klein et al., 2014), so speed- or direction-dependent eye movements induced by stimulus motion would affect pRF estimates in speed- and directiondependent ways. However, we do not find speed- or directiondependence in the eye movements we record. Furthermore, pRF size and eccentricity changes increase up the visual hierarchy, while eye movement effects are similar throughout the hierarchy, and independent of pRF size (Klein et al., 2014).

The slowest speed tested $\left(1.25^{\circ} / \mathrm{s}\right)$ yields larger and more eccentric pRFs than some faster speeds (most clearly $2.5^{\circ} / \mathrm{s}$ ). It may simply be that neurons preferring $1.25^{\circ} / \mathrm{s}$ stimuli have their receptive fields displaced more than neurons preferring some higher speeds. However, this does not lead to a larger perceptual displacement at $1.25^{\circ} / \mathrm{s}$, and an alternative interpretation is possible. Different channels (populations of neurons) process fast and slow motions (van de Grind et al., 1986, 2001; Verstraten et al., 1998). Larger effects on pRF preferred positions and size for $1.25^{\circ} / \mathrm{s}$ stimuli may reflect activity in the slow channel or in non-motion-selective neural populations, that are more sensitive to lower temporal frequencies. We have no experimental evidence to support such speculations. We find no previous reports of either larger receptive field changes with slow motions or very different activation of a specific neural population when motion speed changes from $1.25^{\circ} / \mathrm{s}$ to $2.5^{\circ} / \mathrm{s}$

\section{Mechanism of preferred position changes}

Several mechanisms could produce the observed effects, while other proposed mechanisms may be excluded by our results.
Most simply, preferred position changes may result from greater responses to appearing than continuing motions. Motion onsets produce strong transient responses (Egelhaaf and Borst, 1989; Egelhaaf et al., 1989). Several reports describe larger BOLD responses to appearing than continuing motions (Whitney et al., 2003; Liu et al., 2006; Raemaekers et al., 2009; Schellekens et al., 2013; Maloney et al., 2014). Later visual processing integrates responses from a region of early visual cortex (Maunsell and McAdams, 2000; Motter, 2009; Harvey and Dumoulin, 2011; Haak et al., 2012). An integrating neuron whose inputs represent both the location of appearing motion (with its higher response gain) and any other stimulated location will respond to the position of appearing motion more strongly than the other stimulated location (Figs. 11A \& B). This moves the position that produces the greatest response in the $\mathrm{pRF}$ (the preferred position) towards the appearing motion (Maunsell and McAdams, 2000), against the direction of motion. As this neural activity would normally result from stimuli further along the motion trajectory, perceived position is displaced in the direction of motion.

Motion-induced displacement of perceived position predictively updates target position, and so achieves predictive coding (Roach et al., 2011; Maloney et al., 2014; Schellekens et al., 2014). While low-level mechanisms alone may explain our results, predictive coding may be necessary to explain other effects ((Roach et al., 2011); but see also (Arnold et al., 2014)). Even if predictions arise from low-level mechanisms, changing position preferences may predict future visual stimulation based on experience.

However, accurate prediction of moving objects' positions is inconsistent with our perceptual effects. Asymmetries between motion towards and away from fixation, contrast effects, and speed-independent effects all distinguish perceived position from accurate predictions.

Feedback from MT + has also been proposed to underlie perceived position changes (De Valois and De Valois, 1991; Nishida and Johnston, 1999). Such feedback could indeed amplify responses to appearing motions. However, the effects we find in TO-1 are a similar proportion of pRF size to effects elsewhere. So the representation of space in MT + (Maus et al., 2013) may not have a special role. Therefore, feedback effects are not necessary to explain our results.

Attention could also produce the observed effects (Baldo and Klein, 1995). However, Baldo and Klein proposed that attentional tracking along the continuing motion trajectory beyond the aperture would increase responses to continuing motions. This would displace position
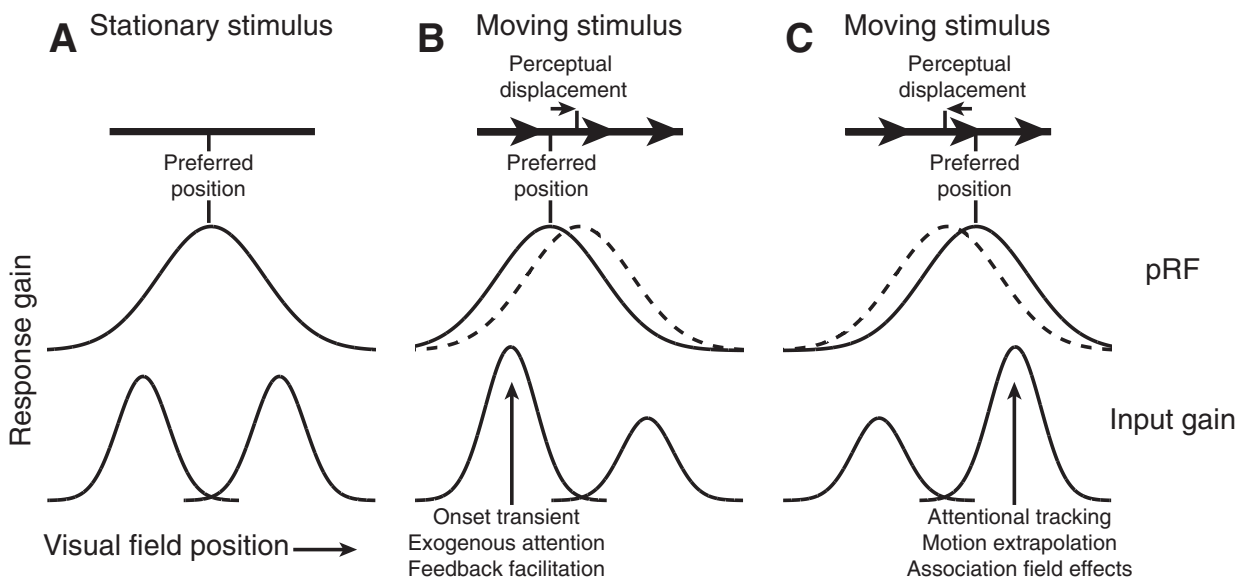

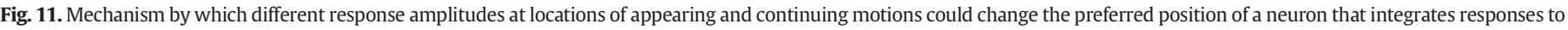

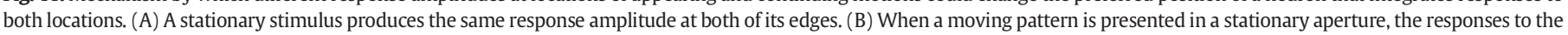

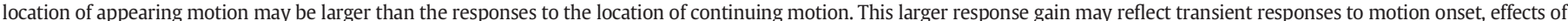

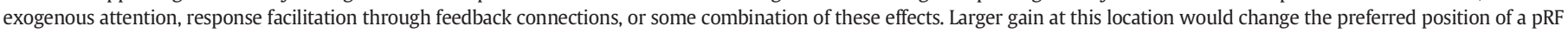

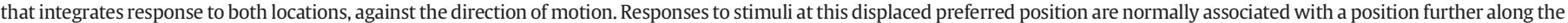

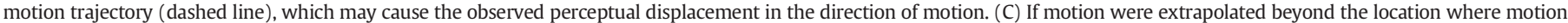

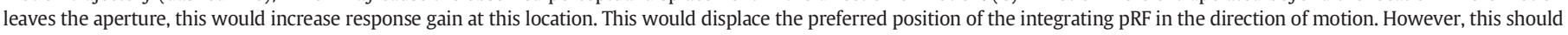
cause perceptual displacements against the direction of motion, which are not seen. 
preferences in the direction of motion (Fig. 11C), and so perceptually displace stimuli in the opposite direction to that observed.

However, endogenous attention affects spatial response preferences as early as V1, moving pRF preferred positions towards attended locations (Klein et al., 2014). Appearing motion is salient and may attract exogenous attention and thereby increase responses to appearing motions (Li, 2002). We cannot distinguish attention from other effects that increase responses to appearing motions.

Finally, extraclassical 'association field' effects may facilitate neural responses along the motion trajectory beyond the stimulus aperture (Watamaniuk and McKee, 1995; Ledgeway and Hess, 2002; Ledgeway et al., 2005). As with attentional tracking, this should increase response amplitudes to continuing motions and displace stimuli against the direction of motion. While we cannot reconcile such effects with perceptual displacements or changes in pRF preferred position, they may contribute to observed pRF size changes.

This proposed mechanism therefore relies on an increase in gain on the pRF's inputs at the stimulus aperture edge where motion appears. Several plausible mechanisms may cause this increase, and are not mutually exclusive. As subsequent stages of the hierarchy integrate spatially separate responses, these early input gain effects will change the most responsive positions in later processing stages. Importantly, we do not propose specific structures to represent these earlier stages (Fig. 11's 'input gain') and later stages (the 'pRF'). We describe input gain effects because they must occur by the input axonal synapses of the population whose pRFs we measure. However, these gain changes could occur earlier. Integration of visual information in the visual hierarchy results from successive stages of increasing spatial integration. This proposed mechanism might act anywhere where such integration occurs, potentially at many layers of the visual hierarchy.

In sum, our results extend effects of visual motion on neural response amplitudes and perceived position to effects on neural position preferences throughout the visual hierarchy. We show that different effect sizes in different visual field maps are proportional to pRF size differences between maps, suggesting that motion transforms spatial response preferences similarly throughout the hierarchy. We propose biologically plausible mechanisms that could produce these changes, linking effects on the neural representation of visual space to the underlying neural architecture.

\section{Acknowledgments}

This work was supported by Netherlands Organization for Scientific Research grants \#452.08.008 to SD and \#433.09.223 to SD and FW Cornelissen, and by Portuguese Foundation for Science and Technology grant \#IF/01405/2014 to BH.

\section{References}

Amano, K., Wandell, B.A., Dumoulin, S.O., 2009. Visual field maps, population receptive field sizes, and visual field coverage in the human MT + complex. J. Neurophysiol. 102, 2704-2718

Arnold, D.H., Marinovic, W., Whitney, D., 2014. Visual motion modulates pattern sensitivity ahead, behind, and beside motion. Vis. Res. 98, 99-106.

Baldo, M.V., Klein, S.A., 1995. Extrapolation or attention shift? Nature 378, 565-566.

Brainard, D.H., 1997. The psychophysics toolbox. Spat. Vis. 10, 433-436.

Culham, J.C., Dukelow, S.P., Vilis, T., Hassard, F.A., Gati, J.S., Menon, R.S., Goodale, M.A., 1999. Recovery of fMRI activation in motion area MT following storage of the motion aftereffect. J. Neurophysiol. 81, 388-393.

Dale, A.M., Fischl, B., Sereno, M.I., 1999. Cortical surface-based analysis. I. Segmentation and surface reconstruction. NeuroImage 9, 179-194.

De Valois, R.L., De Valois, K.K., 1991. Vernier acuity with stationary moving Gabors. Vis. Res. 31, 1619-1626.

Dumoulin, S.O., Wandell, B.A., 2008. Population receptive field estimates in human visual cortex. Neurolmage 39, 647-660.

Dumoulin, S.O., Hess, R.F., May, K.A., Harvey, B.M., Rokers, B., Barendregt, M., 2014. Contour extracting networks in early extrastriate cortex. J. Vis. 14, 18.

Egelhaaf, M., Borst, A., 1989. Transient and steady-state response properties of movement detectors. J. Opt. Soc. Am. A Opt. Image Sci. 6, 116-127.
Egelhaaf, M., Borst, A., Reichardt, W., 1989. Computational structure of a biological motion-detection system as revealed by local detector analysis in the fly's nervous system. J. Opt. Soc. Am. A Opt. Image Sci. 6, 1070-1087.

Fischer, J., Spotswood, N., Whitney, D., 2011. The emergence of perceived position in the visual system. J. Cogn. Neurosci. 23, 119-136.

Friston, K.J., Fletcher, P., Josephs, O., Holmes, A., Rugg, M.D., Turner, R., 1998. Event-related fMRI: characterizing differential responses. Neurolmage 7, 30-40.

Frund, I., Haenel, N.V., Wichmann, F.A., 2011. Inference for psychometric functions in the presence of nonstationary behavior. J. Vis. 11.

Fu, Y.X., Shen, Y., Gao, H., Dan, Y., 2004. Asymmetry in visual cortical circuits underlying motion-induced perceptual mislocalization. J. Neurosci. 24, 2165-2171.

Glover, G.H., 1999. Deconvolution of impulse response in event-related BOLD fMRI Neurolmage 9, 416-429.

Haak, K.V., Winawer, J., Harvey, B.M., Renken, R., Dumoulin, S.O., Wandell, B.A., Cornelissen, F.W., 2012. Connective field modeling. Neurolmage 66C, 376-384.

Harvey, B.M., Dumoulin, S.O., 2011. The relationship between cortical magnification factor and population receptive field size in human visual cortex: constancies in cortical architecture. J. Neurosci. 31, 13604-13612.

Harvey, B.M., Klein, B.P., Petridou, N., Dumoulin, S.O., 2013. Topographic representation of numerosity in the human parietal cortex. Science 341, 1123-1126.

He, S., Cohen, E.R., Hu, X., 1998. Close correlation between activity in brain area MT/V5 and the perception of a visual motion aftereffect. Curr. Biol. 8, 1215-1218.

Hoffmann, M.B., Kaule, F.R., Levin, N., Masuda, Y., Kumar, A., Gottlob, I., Horiguchi, H., Dougherty, R.F., Stadler, J., Wolynski, B., Speck, O., Kanowski, M., Liao, Y.J., Wandell, B.A., Dumoulin, S.O., 2012. Plasticity and stability of the visual system in human achiasma. Neuron 75, 393-401.

Huk, A.C., Ress, D., Heeger, D.J., 2001. Neuronal basis of the motion aftereffect reconsidered. Neuron 32, 161-172.

Klein, B.P., Harvey, B.M., Dumoulin, S.O., 2014. Attraction of position preference by spatial attention throughout human visual cortex. Neuron 84, 227-237.

Larsson, J., Heeger, D.J., 2006. Two retinotopic visual areas in human lateral occipital cortex. J. Neurosci. 26, 13128-13142.

Ledgeway, T., Hess, R.F., 2002. Rules for combining the outputs of local motion detectors to define simple contours. Vis. Res. 42, 653-659.

Ledgeway, T., Hess, R.F., Geisler, W.S., 2005. Grouping local orientation and direction signals to extract spatial contours: empirical tests of "association field" models of contour integration. Vis. Res. 45, 2511-2522.

Levin, N., Dumoulin, S.O., Winawer, J., Dougherty, R.F., Wandell, B.A., 2010. Cortical maps and white matter tracts following long period of visual deprivation and retinal image restoration. Neuron $65,21-31$.

Li, Z., 2002. A saliency map in primary visual cortex. Trends Cogn. Sci. 6, 9-16.

Liu, J.V., Ashida, H., Smith, A.T., Wandell, B.A., 2006. Assessment of stimulus-induced changes in human V1 visual field maps. J. Neurophysiol. 96, 3398-3408.

Maloney, R.T., Watson, T.L., Clifford, C.W., 2014. Determinants of motion response anisotropies in human early visual cortex: the role of configuration and eccentricity NeuroImage 100, 564-579.

Maunsell, J.H.R., McAdams, C.J., 2000. Effects of attention on neural response properties in visual cerebral cortex. In: Gazzaniga, M.S. (Ed.), The New Cognitive Neurosciences. MIT Press, pp. 315-324.

Maus, G.W., Fischer, J., Whitney, D., 2013. Motion-dependent representation of space in area MT +. Neuron 78, 554-562.

Motter, B.C., 2009. Central V4 receptive fields are scaled by the V1 cortical magnification and correspond to a constant-sized sampling of the V1 surface. J. Neurosci. 29, 5749-5757.

Nestares, O., Heeger, D.J., 2000. Robust multiresolution alignment of MRI brain volumes Magn. Reson. Med. 43, 705-715.

Nishida, S., Johnston, A., 1999. Influence of motion signals on the perceived position of spatial pattern. Nature 397, 610-612.

Olman, C.A., Inati, S., Heeger, D.J., 2007. The effect of large veins on spatial localization with GE BOLD at 3 T: displacement, not blurring. NeuroImage 34, 1126-1135.

Pelli, D.G., 1997. The VideoToolbox software for visual psychophysics: transforming numbers into movies. Spat. Vis. 10, 437-442.

Raemaekers, M., Lankheet, M.J., Moorman, S., Kourtzi, Z., van Wezel, R.J., 2009. Directional anisotropy of motion responses in retinotopic cortex. Hum. Brain Mapp. 30, 3970-3980.

Ramachandran, V.S., Anstis, S.M., 1990. Illusory displacement of equiluminous kinetic edges. Perception 19, 611-616.

Roach, N.W., McGraw, P.V., Johnston, A., 2011. Visual motion induces a forward prediction of spatial pattern. Curr. Biol. 21, 740-745.

Schellekens, W., Van Wezel, R.J., Petridou, N., Ramsey, N.F., Raemaekers, M., 2013. Integration of motion responses underlying directional motion anisotropy in human early visual cortical areas. PLoS One 8, e67468.

Schellekens, W., van Wezel, R.J., Petridou, N., Ramsey, N.F., Raemaekers, M., 2014. Predictive coding for motion stimuli in human early visual cortex. Brain Struct. Funct.

Sereno, M.I., Dale, A.M., Reppas, J.B., Kwong, K.K., Belliveau, J.W., Brady, T.J., Rosen, B.R. Tootell, R.B., 1995. Borders of multiple visual areas in humans revealed by functional magnetic resonance imaging. Science 268, 889-893.

Smith, A.T., Singh, K.D., Williams, A.L., Greenlee, M.W., 2001. Estimating receptive field size from fMRI data in human striate and extrastriate visual cortex. Cereb. Cortex $11,1182-1190$.

Sundberg, K.A., Fallah, M., Reynolds, J.H., 2006. A motion-dependent distortion of retinotopy in area V4. Neuron 49, 447-457.

Swisher, J.D., Halko, M.A., Merabet, L.B., McMains, S.A., Somers, D.C., 2007. Visual topography of human intraparietal sulcus. J. Neurosci. 27, 5326-5337.

Teo, P.C., Sapiro, G., Wandell, B.A., 1997. Creating connected representations of cortical gray matter for functional MRI visualization. IEEE Trans. Med. Imaging 16 852-863. 
van de Grind, W.A., Koenderink, J.J., van Doorn, A.J., 1986. The distribution of human motion detector properties in the monocular visual field. Vis. Res. 26, 797-810.

van de Grind, W.A., van Hof, P., van der Smagt, M.J., Verstraten, F.A., 2001. Slow and fast visual motion channels have independent binocular-rivalry stages. Proc. Biol. Sci. $268,437-443$

Verstraten, F.A., van der Smagt, M.J., van de Grind, W.A., 1998. Aftereffect of high-speed motion. Perception 27, 1055-1066.

Wandell, B.A., Chial, S., Backus, B.T., 2000. Visualization and measurement of the cortical surface. J. Cogn. Neurosci. 12, 739-752.

Wandell, B.A., Dumoulin, S.O., Brewer, A.A., 2007. Visual field maps in human cortex. Neuron 56, 366-383.

Watamaniuk, S.N., McKee, S.P., 1995. Seeing motion behind occluders. Nature 377, 729-730.

Whitney, D., 2002. The influence of visual motion on perceived position. Trends Cogn. Sci. $6,211-216$
Whitney, D., Goltz, H.C., Thomas, C.G., Gati, J.S., Menon, R.S., Goodale, M.A., 2003. Flexible retinotopy: motion-dependent position coding in the visual cortex. Science 302, $878-881$.

Winawer, J., Horiguchi, H., Sayres, R.A., Amano, K., Wandell, B.A., 2010. Mapping hV4 and ventral occipital cortex: the venous eclipse. J. Vis. 10.

Worsley, K.J., Liao, C.H., Aston, J., Petre, V., Duncan, G.H., Morales, F., Evans, A.C., 2002. A general statistical analysis for fMRI data. NeuroImage 15, 1-15.

Yushkevich, P.A, Piven, J., Hazlett, H.C., Smith, R.G, Ho, S, Gee, J.C, Gerig, G, 2006. User-guided 3D active contour segmentation of anatomical structures: significantly improved efficiency and reliability. NeuroImage 31, 1116-1128.

Zuiderbaan, W., Harvey, B.M., Dumoulin, S.O., 2012. Modeling center-surround configurations in population receptive fields using fMRI. J. Vis. 12 (3), 10. 